\title{
Simulationen von Strömungsakustik in rotierenden Bauteilen zur Entwicklung von Antriebskonzepten der Autos der Zukunft
}

\author{
C. Freidhager, P. Maurerlehner, K. Roppert, A. Wurzinger, A. Hauser, M. Heinisch, S. Schoder, M. Kaltenbacher
}

Die Antriebstechniken der Zukunft, wie z. B. der Elektromotor oder die Brennstoffzelle, erzeugen selbst nur sehr wenig Lärm, wodurch die Akustik der Hilfsaggregate in den Vordergrund tritt. Speziell rotierende Systeme wie die Kühlventilatoren des Elektromotors oder der Turbolader der Brennstoffzelle sind dabei Hilfsaggregate, denen eine große Bedeutung zukommt. Messungen an großen Versuchsaufbauten, um die Mechanismen, die in diesen Systemen Lärm verursachen zu verstehen, sind dabei häufig zu kosten- und zeitintensiv. Demzufolge werden immer häufiger komplexe Simulationen verwendet, um tiefergehendes Wissen über die Strömungsakustik in rotierenden Systemen aufzubauen. In diesem Beitrag präsentieren wir dazu eine Simulationsmethodik und zwei Anwendungen aus der Automobiltechnik.

Schlüsselwörter: Strömungsakustik; Simulation; Brennstoffzelle; Verdichter; Kühlung; Ventilatoren; Batteriekühlung; HVAC

\begin{abstract}
Aeroacoustic simulations applied to rotating components of future power units of cars.
Future power units of cars, e.g., the electric engine or the fuel cell, generate little noise. Consequently, the noise connected to auxiliary units becomes more dominant. Thereby, especially rotating components are of great importance, e.g., the cooling fan of a battery unit or the turbocharger of fuel cells. Since performing complex measurements are expensive and require a lot of time, simulations enhance current development processes. Hence, we present two different aeroacoustic simulation approaches applied to two examples of the automotive industry.
\end{abstract}

Keywords: aeroacoustic; simulation; fuel cell; compressor; ventilation; fan; battery cooling; HVAC

Eingegangen am 26. Jänner 2021, angenommen am 30. März 2021, online publiziert am 7. April 2021

(C) The Author(s) 2021

\begin{abstract}
1. Einleitung
Strömungsakustische Untersuchungen werden in vielen verschiedenen Industriezweigen durchgeführt und beschäftigen sich mit akus tischen Phänomenen die in Verbindung mit Strömungen auftreten. Bei diesem Forschungsgebiet handelt es sich also um eine Kombination aus Strömungsmechanik und Akustik. Speziell im Bereich der Automobiltechnik sind dabei vielfach rotierende Systeme wie Ventilatoren, oder Turbolader für auftretenden Schall, der als Lärm empfunden wird, verantwortlich. Vor allem bei Antriebstechniken der Zukunft, wie z. B. dem Elektromotor, oder der Brennstoffzelle, bei denen der eigentliche Motor keine wesentlichen, kaschierende Geräusche erzeugt, wird dies zum Problem. Um solche Lärmquellen untersuchen zu können werden zeit- und kostenintensive Messungen und Experimente durchgeführt. Begründet durch das Bestreben nach immer kürzeren Entwicklungszeiten und immer komplexeren Funktionsgruppen, sind die dafür notwendigen, aufwendigen Versuchsaufbauten für den Entwicklungsprozess kein adäquates Hilfsmittel mehr um strömungsakustische Phänomene zu verstehen. Zusätzlich besteht bei Experimenten das Problem, dass viele strömungsakustische Größen messtechnisch nicht zugänglich sind, oder nicht unmittelbar erfasst werden können. Eine Alternative dazu bieten numerische Verfahren, die präzise Computersimulation von Strömungsakustik ermöglichen. Folglich gewinnen strömungsakustische Simulationen an Bedeutung [11], auch weil sie die Untersuchung von physikalischen Effekten (wie z. B. akustischen Quelltermmechanismen) erlauben, die nicht unmittelbar mit Messergebnissen
\end{abstract}

abgebildet werden können. Außerdem ermöglichen Simulationen das Durchführen von relativ schnellen Optimierungen von Bauteilen und Komponenten. Der verbleibende Nutzen von Messungen besteht in der Validierung von Simulationskonfigurationen und Methodiken. In den aller meisten Fällen beruhen numerische Simulationen auf Modellen, denen zu Grunde verschiedenste Annahmen liegen und die dem zur Folge die Realität nur approximativ beschreiben. Somit bilden Simulationsergebnisse, abhängig vom gewählten Simulationsmodell, die reale Physik oftmals nur näherungsweise ab. Die Wahl des Simulationsmodells und der Simulationsmethodik ist damit abhängig von der Untersuchung die durchgeführt werden soll. Ein weiterer Faktor in diesem Auswahlprozess sind die zur Verfügung stehenden Rechenressourcen. Obwohl Rechenzentren in den vergangenen Jahrzehnten massiv an Leistungsfähigkeit zugelegt haben (wie z. B. die Supercomputer des Vienna Scientific Cluster [12]), ist die Wahl der Simulationsmethodik noch immer sehr stark durch

Freidhager, Clemens, Institut für Grundlagen und Theorie der Elektrotechnik, Inffeldgasse 18/, 8010 Graz, Österreich (E-Mail: clemens.freidhager@tugraz.at); Maurerlehner, Paul, Institut für Grundlagen und Theorie der Elektrotechnik, Graz, Österreich; Roppert, Klaus, Institut für Grundlagen und Theorie der Elektrotechnik, Graz, Österreich; Wurzinger, Andreas, Institut für Grundlagen und Theorie der Elektrotechnik, Graz, Österreich; Hauser, Alexander, Institut für Mechanik und Mechatronik, Technische Universität Wien, Wien, Österreich; Heinisch, Martin, BMW Motoren GmbH Steyr, Steyr, Österreich; Schoder, Stefan, Institut für Grundlagen und Theorie der Elektrotechnik, Graz, Österreich; Kaltenbacher, Manfred, Institut für Grundlagen und Theorie der Elektrotechnik, Graz, Österreich 


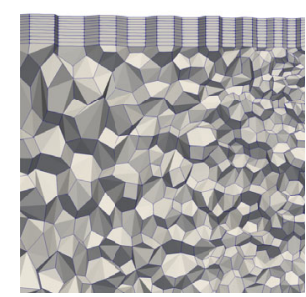

(a) Strömungsgitter

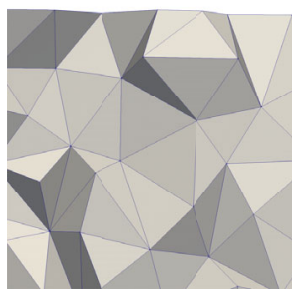

(b) Akustikgitter

Abb. 1. Unterschiedliche Simulationsgitter zur Approximierung des Simulationsgebietes. Abbildung 1a zeigt ein exemplarisches Strömungsgitter und Abb. $1 \mathrm{~b}$ ein dazugehöriges exemplarisches Akustikgitter. Das Netz zur Strömungssimulation ist dabei durch Polyedra und prismaförmige Elemente gekennzeichnet, das Akustiknetz durch tetraederförmige Elemente

die nur begrenzt zur Verfügung stehenden Rechenressourcen beeinflusst.

Um Strömungsakustik zu beschreiben, bedient man sich partiellen Differentialgleichungen, die numerisch gelöst werden. Bei dem von uns präsentierten Ansatz unterscheidet man dabei zwischen den Gleichungen, die das Strömungsfeld beschreiben und den Gleichungen die das akustische Feld beschreiben. Die Gleichungen des Strömungsfeldes werden dabei häufig mit dem Finite Volumen (FV) Verfahren und die Gleichungen die das akustische Feld beschreiben mit der Finiten Elemente Methode (FEM) [2] gelöst. Beide Verfahren, FV und FEM, beruhen dabei auf Approximationen des Simulationsgebietes, auf einem sogenannten Rechengitter. Für Simulationen des akustischen Feldes wird die mMultiphysics-Software openCFS verwendet [14].

Die unterschiedlichen Simulationsverfahren, zur Berechnung von Strömung und Akustik, haben dabei unterschiedliche Anforderungen an das Rechengitter (siehe Abb. 1), was einen Grund darstellt, warum in der Simulationsmethodik die Berechnung von Strömung und Akustik voneinander getrennt wird. Diese Methodik wird dabei auch als hybrider Simulationsansatz bezeichnet [1]. Im nachfolgenden Beitrag wird auf die Herausforderungen von Strömungsakustiksimulationen in Verbindung mit rotierenden Systemen und dem hybriden Simulationsansatz eingegangen. Dabei werden unterschiedliche strömungsakustische Analogien präsentiert, die zur Kopplung des Strömungs- und Akustikfeldes verwenden werden. Abschließend werden zwei Anwendungsbeispiele besprochen.

\section{Hybrider strömungsakustischer Simulationsansatz}

Der hybride strömungsakustische Simulationsansatz besteht aus drei unterschiedlichen Arbeitschritten, welche chronologisch abgearbeitet werden müssen und die einander nicht überschneiden (siehe Abb. 2).

\subsection{Strömungssimulation}

Als erster Schritt wird eine Strömungssimulation durchgeführt. Diese Simulation ist zeitabhängig und beruht auf der Definition einer Zeit-

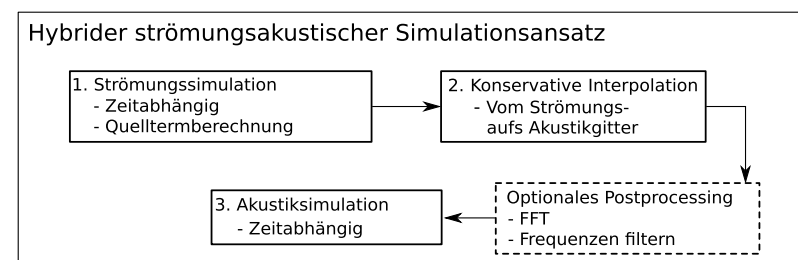

Abb. 2. Drei Hauptschritte des hybriden strömungsakustischen Simulationsansatz

schrittweite die sich an den zu untersuchenden Phänomenen orientiert. Je nach der zu untersuchenden Strömung müssen die kompressiblen oder inkompressiblen Strömungsgleichungen gelöst werden. Bei Berechnungen mit rotierenden Systemen wird zusätzlich das Netz der rotierenden Regionen während der Simulation um einen definierten Winkelschritt pro Zeitschritt weiter gedreht, um den Einfluss der Rotation abbilden zu können. Im Zuge einer solchen Strömungssimulation werden auf Basis einer strömungsakustischen Analogie (siehe Kap. 2.4) akustische Quellterme berechnet. Eine strömungsakustische Analogie stellt dabei eine Kopplungsstrategie von Strömungs- und Akustikfeld dar. Da der Strömungssimulation häufig die Finite Volumen Methode zugrunde liegt, gibt es für jede Zelle im Strömungsnetz einen Quelltermwert. Jeder dieser Quelltermwerte wird für jeden Zeitschritt exportiert, wodurch sehr große Datenmengen erzeugt werden. Da die Daten von mehreren Umdrehungen exportiert werden müssen, um in weiterer Folge niedrigfrequente Phänomene untersuchen zu können, benötigt das Exportieren von Quelltermen sehr schnell einige Terabyte an Speicherplatz. Zur Simulation von Strömungen werden Programme wie z. B. StarCCM+, Fluent, oder auch OpenFOAM verwendet.

\subsection{Interpolation}

Als zweiter Schritt werden die zuvor berechneten strömungsakustischen Quellterme energieerhaltend [15] vom Strömungs- auf das Akustiknetz interpoliert. Unter Interpolation versteht man dabei das Überführen einer Größe wie, z. B. des Quellterms, von einem Simulationsnetz auf ein anderes. Da die beiden Netze aus geometrisch unterschiedlichen Elementen bestehen und diese unterschiedlich groß sein können (siehe Abb. 1) werden hierfür spezielle Algorithmen verwendet. Diese Algorithmen verschneiden die unterschiedlichen Elemente des Strömungs- und Akustiknetzes und gewichten die Quelltermgrößen mit den daraus resultierenden Verschnittvolumina. Durch diese Interpolationsmethode bleibt die Energie der Quellterme lokal und global erhalten [13]. Außerdem werden die Quellterme am Akustiknetz nicht mehr den Zellen, sondern den Knoten, die die Zellen beschreiben, zugeordnet. Dies ist notwendig, weil die nachfolgende Akustiksimulation auf der knotenbasierten Finite Elemente Methode [2] beruht. Für die Interpolation wird das Datenverarbeitungstool [3] von openCFS [14] verwendet.

\subsection{Akustiksimulation}

Um Akustiksimulationen, auch Ausbreitungsrechnungen genannt, durchführen zu können wird, die inhomogene Wellengleichung [2]

$$
\left(\frac{1}{c_{0}^{2}} \frac{D^{2}}{D t^{2}}-\nabla \cdot \nabla\right) p^{\prime}=S_{\text {Quellterm }}
$$

verwendet. Dabei bezeichnet $c_{0}$ die mitllere Schallgeschwindigkeit bei Umgebungsbedingungen (bei $20^{\circ} \mathrm{C}$ in etwa $341 \mathrm{~m} / \mathrm{s}$ ), $\nabla$ den Nablaoperator, $p^{\prime}$ die Schwankungsgröße des Drucks und SQuellterm 


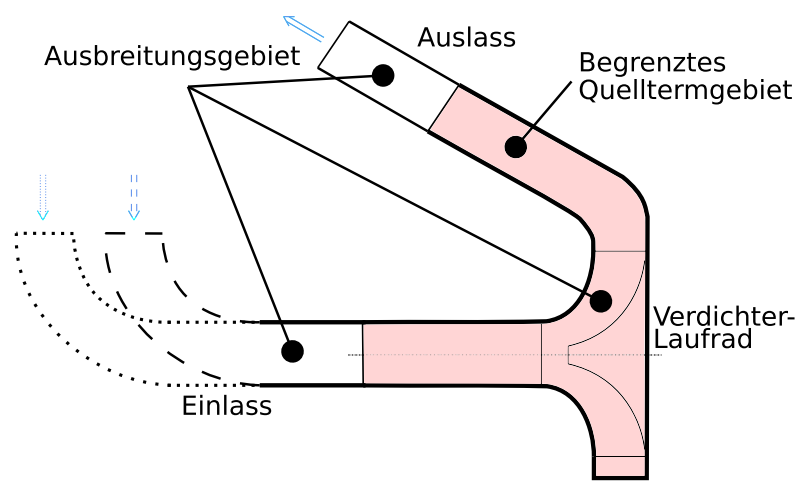

Abb. 3. Begrenztes Quelltermgebiet (dargestellt in rot) und größeres Ausbreitungsgebiet mit unterschiedlichen Einlassgeometrien, dargestellt anhand eines schematischen Turboladerverdichters. Die Strömungsrichtungen im Bereich des Verdichterein- und Auslasses sind als blaue Pfeile dargestellt

den Quellterm, der basierend auf einer strömungsakustischen Analogie im Zuge der Strömungssimulation berechnet wird. Die Wellengleichung wird FEM-basiert numerisch gelöst, wobei entsprechende Randbedingungen aufgeprägt werden müssen [2]. Randbedingungen begrenzen das Simulationsgebiet, z. B. im Bereich von Wänden. Speziell wenn in der Realität unbegrenzte Gebiete, wie z. B. ein Ventilator der Luft aus der Umgebung ansaugt, simuliert werden müssen, ist eine Reduktion des Simulationsgebietes durch spezielle Randbedingungen notwendig. Dafür werden häufig Perfectly Matched Layers [2] (PML) oder auch Absorbing Boundary Conditions [2] $(A B C)$ verwendet. Die Ausbreitungsrechnung wird dabei ebenfalls mit openCFS durchgeführt.

Der hybride Simulationsansatz bietet also die Möglichkeit, ein optimiertes Strömungsnetz und ein optimiertes Akustiknetz zu verwenden. Zusätzlich erlaubt der Ansatz das Gebiet von Strömungssimulationen auf kleine Bereiche zu begrenzen in denen Quellterme auftreten (siehe Abb. 3). Dadurch kann der Rechenaufwand für die zum Teil sehr aufwendigen Strömungssimulationen massiv reduziert werden. Die Ausbreitungsrechnung findet anschließend wieder für das gesamte Gebiet, dem Ausbreitungsgebiet, statt. Folglich ermöglicht der Ansatz das einfache variieren von unterschiedlichen Geometrien des Ausbreitungsgebietes sowie das Bewerten unterschiedlicher Absorberstrukturen, solange dies nicht die eigentlichen Quellterme beeinflusst. Dies ist möglich, da die Strömungssimulation und die Quelltermberechnung den aufwendigsten Teil der Methodik darstellt und diese nur einmalig durchgeführt werden muss.

Außerdem bietet openCFS die Möglichkeit z. B. ein akustisches Feld mit einem mechanischen Feld zu koppeln. Dabei kann die oben skizzierte Ausbreitungsrechnung verwendet werden, um durch eine Kopplung mit der mechanischen Struktur, z. B. Körperschall (also Schwingungen der Struktur), zu untersuchen. Solche Kopplungen können ebenfalls mehrfach angewendet werden, um beispielsweise basierend auf einer Ausbreitungsrechnung in einem Rohr, den abgestrahlten Schall zu simulieren. Dafür sind zwei Kopplungen, eine zwischen Akustik im Rohr und mechanischer Struktur, und einer zwischen mechanischer Struktur und Akustik außerhalb des Rohrs notwendig.

\subsection{Strömungsakustische Analogien}

In weiterer Folge werden eine strömungsakustische Analogie und eine strömungsakustische Formulierung und ihre entsprechenden
Vor- und Nachteile präsentiert. Beide Ansätze können dabei im Zeitbereich oder nach einer Fourier-Transformation (FT) im Frequenzbereich durchgeführt werden. Eine Simulation im Zeitbereich bedeutet, dass die Differentialgleichungen für einen Zeitpunkt nach dem anderen gelöst werden eine Simulation im Frequenzbereich, dass die Differentialgleichungen für verschiedene Frequenzen gelöst werden. Das bedeutet, im Frequenzbereich können auch nur einzelne Frequenzen betrachtet werden. Beide Ansätze haben dabei verschiedene Vor- und Nachteile, wobei anzumerken ist, dass die Berücksichtigung rotierender Maschinenteile nur im Zeitbereich möglich ist.

\subsubsection{Lighthill Analogie}

Die Lighthill Analogie $[9,10]$ beruht auf dem Vergleich von einem fluktuierenden Fluid und einem Fluid in Ruhe, und einer exakten Umformulierungen von Massenerhaltung und Impulserhaltung. Bei Verwendung der Lighthill Analogie werden sämtliche aeroakustische und aerodynamische Druckschwankungen beschrieben. Folglich ist es also nicht ohne weiteres möglich, reine akustische Druckschwankungen zu untersuchen. Lediglich in ausreichend großem Abstand von dem Quelltermgebiet, wo es zu keinen aerodynamischen Druckschwankungen mehr kommt, können akustische Druckschwankungen untersucht werden. Dieses Phänomen ist in der Strömungsakustik unter dem Begriff der Disparität der Skalen bekannt, die beschreibt, dass aerodynamische Druckschwankungen wesentlich schneller abklingen, als akustische Druckschwankungen. Bei Verwendung dieser Analogie ergibt sich ein strömungsakustischer

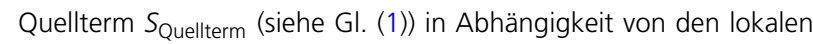
Größen der Strömungsgeschwindigkeit $\boldsymbol{v}$, dem Druck $p$, sowie der Dichte $\rho$

$$
S_{\text {Quellterm }}=\nabla \cdot\left[\nabla \cdot\left(\rho \boldsymbol{v} \otimes \boldsymbol{v}+\left(p^{\prime}-c_{0}^{2} \rho^{\prime}\right)[\boldsymbol{l}]-[\boldsymbol{\tau}]\right)\right] .
$$

Dabei ist [ $\boldsymbol{l}]$ der Einheitstensor und [ $\boldsymbol{\tau}]$ der materialabhängigen viskose Spannungstensor. Eine Beschränkung der Lighthillanalogie hinsichtlich kompressibler/inkompressibler Strömung gibt es dabei nicht. Im Falle einer inkompressiblen Strömung vereinfacht sich jedoch der aeroakustische Quellterm $S_{\text {Quellterm deutlich. Strömungen }}$ werden dabei als inkompressible verstanden, wenn das Verhältnis aus ihrer Geschwindigkeit und der Schallgeschwindigkeit, genannt Machnummer Ma, nicht größer als $M a \leq 0.3$ ist. Eine weitere Einschränkung ist, dass die zuvor präsentierte Wellengleichung (siehe $\mathrm{Gl}$. (1)) für Überschallströmungen nur eingeschränkt gültig ist.

\subsubsection{PCWE}

Diese strömungsakustische Formulierung [2] beruht auf einem Störansatz, dem zur Folge der Druck, Dichte und die Geschwindigkeit in einen zeitlich unveränderlichen Gleichanteil und eine Schwankungsgröße aufgeteilt wird. Die Schwankungsgröße wird anschließend zusätzlich noch in einen akustischen und einen inkompressiblen Teil aufgespalten, wie nachfolgend exemplarisch für den Druck dargestellt

$$
p=\bar{p}+p^{\mathrm{ic}}+p^{\mathrm{a}} .
$$

Die dabei zugrunde liegenden Annahmen erlauben die Anwendung der PCWE nur bei inkompressiblen Strömungen. Der große Vorteil der PCWE ist dabei die Aufspaltung des Drucks, die direkt die akustische Druckschwankung $p^{\text {a }}$ liefert. Folglich erlaubt diese Auftrennung das Untersuchen einer reinen akustischen Druckschwankung auch in Bereichen in denen der akustische und der aerodynamische 


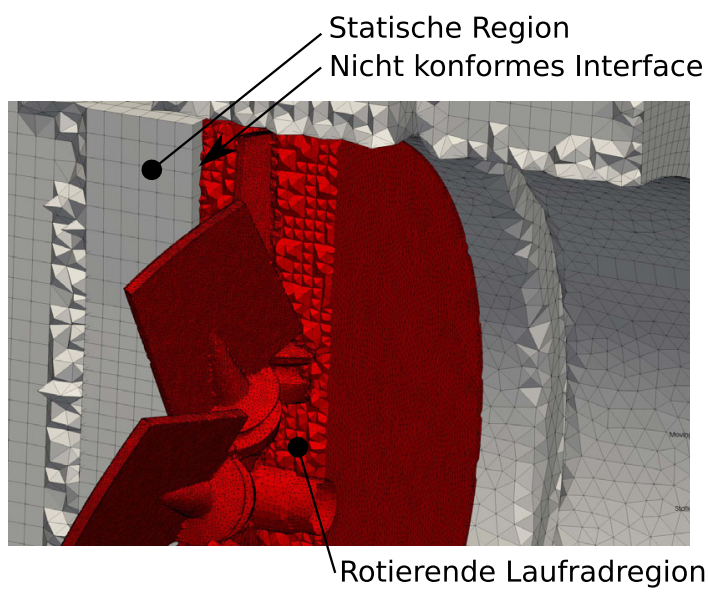

Abb. 4. Nicht konformes Interface zwischen der rotierenden Laufradregion des Verdichterlaufrads (dargestellt in rot) und der statischen Region am Auslass (dargestellt in grau)

Druck überlagert sind. Unter Berücksichtigung eines akustischen Potentials $\psi_{a}$

$$
p^{\mathrm{a}}=\rho_{0} \frac{\partial \psi^{\mathrm{a}}}{\partial t}
$$

wird für diese strömungsakustische Formulierung die Wellengleichung (siehe Gl. (1)) umformuliert und man erhält die substantielle zeitliche Ableitung des inkompressiblen Drucks als Quellterm

$$
S_{\text {Quellterm }}=-\frac{1}{\rho_{0} C_{0}^{2}} \frac{D p^{i c}}{D t} .
$$

\subsection{Berücksichtigung von Rotation}

Die rotatorische Starrkörperbwegung von z. B. einem Ventilatorlaufrad wird zum einen durch die substantielle Zeitableitung

$$
\frac{\mathrm{D}}{\mathrm{D} t}=\frac{\partial}{\partial t}+\left(\overline{\boldsymbol{v}}-\boldsymbol{v}_{\mathrm{r}}\right) \cdot \nabla
$$

berücksichtigt, wobei $\overline{\boldsymbol{v}}$ die mittlere Geschwindigkeit und $\boldsymbol{v}_{\mathrm{r}}$ die Rotationsgeschwindigkeit beschreiben. Zum anderen werden auch für die Ausbreitungsrechnung die rotierenden Regionen gedreht. Durch die Rotation kommt es dabei zu nicht konformen Schnittstellen zwischen den rotierenden und den stillstehenden Regionen des Rechengitters (see Fig. 4). Um an diesen Schnittstellen keine Fehlerquellen für die Simulation zu erhalten, werden nicht konforme Nitsche Interfaces [8] verwendet, die sicherstellen, dass die Schalwelle über die Schnittstellen ungestört propagieren können. Wie zuvor bei den Strömungssimulationen wird auch hierbei das Netz pro definierten Zeitschritt um einen gewissen Betrag weitergedreht. Dies bedeutet, dass die Netze der rotierenden und stillstehenden Regionen nach jedem Zeitschritt in einer neuen relativen Position liegen und somit die Schnittgitter des nicht konformen Nitsche Interfaces neu berechnet werden müssen.

\section{Anwendungen in der Automobiltechnik}

Die Automobilindustrie befindet sich gegenwärtigen in einem radikalen Wandel, hervorgerufen durch die Dringlichkeit der Klimakatastrophe und die dadurch zu implementierenden neuen Antriebskonzepte. Darüber hinaus werden auch die bestehenden Technologie der Verbrennungsmotoren verbessert, um die klimaschädliche $\mathrm{CO}_{2}$-Emissionen zu reduzieren. Diese Entwicklungstrends führen zu einer Vielzahl an Herausforderungen, wobei einige im Bereich

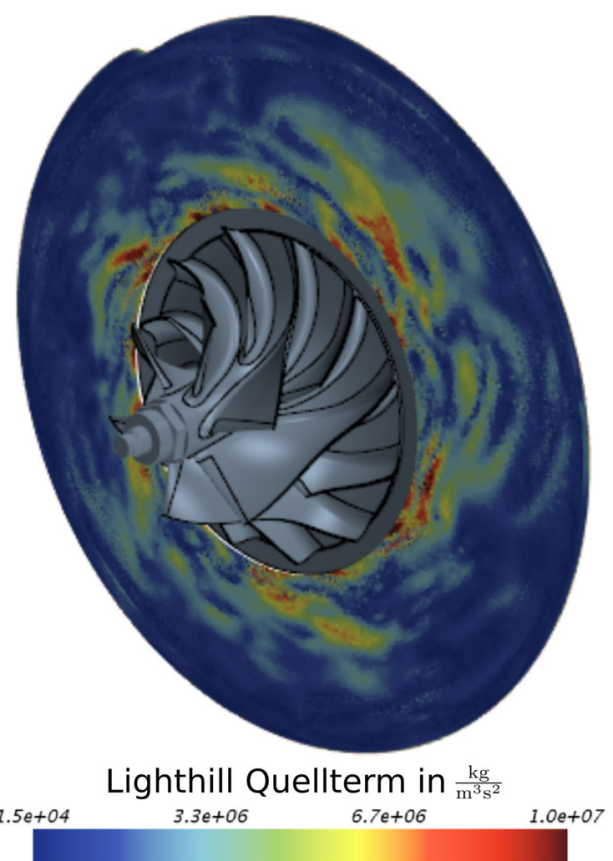

Abb. 5. Lighthill Quellterme im Auslass des Turboladerverdichters

der Strömungsakustik zu finden sind. Da moderne Antriebskonzepte, wie z. B. ein Elektromotor, deutlich geräuschärmer sind, treten strömungsakustische Phänomene immer mehr in den Vordergrund. Nachfolgend werden zwei praktisch relevante Anwendungsbeispiele diskutiert, welche mit der zuvor präsentierten Simulationsmethode analysiert werden.

3.1 Turbolader in Verbrennungsmotor sowie Brennstoffzelle Turbolader spielen eine zentrale Rolle bei der Optimierung von Verbrennungsmotoren durch sogenanntes Downsizing [6]. Da der Lärm, der unmittelbar mit dem Verbrennungsmotor in Verbindung steht, durch elektrische Einspritzsysteme in den letzten Jahren deutlich reduziert werden konnte, tritt der Turbolader akustisch mehr in den Vordergrund. Darüber hinaus kommen Turbolader auch in Kombination mit Brennstoffzellen zur Anwendung, welche überhaupt keine kaschierenden Geräusche produzieren. Als Hauptlärmquelle im Turbolader gilt der Verdichter [7], weswegen in der nachfolgenden Untersuchung die Turboladerturbine vernachlässigt wird. Folglich wird in diesem Anwendungsbeispiel ein Radialturboladerverdichter mit einem maximalen Durchmesser von $82 \mathrm{~mm}$ beim Verdichterlaufrad untersucht. Die untersuchte Drehzahl beträgt dabei 120000 Umdrehungen pro Minute, woraus sich lokale Machzahlen von $M a \geq 1$ innerhalb des Verdichterlaufrads ergeben. Es kommt also lokal zu Überschallströmungen. Somit muss die Strömungssimulation kompressibel ausgeführt werden. Dem zur Folge wird zur Berechnung der Quellterme die Lighthill Analogie verwendet (siehe Abb. 5). Das Gitter für die Strömungssimulation besteht aus 15 Millionen Zellen und auf 160 Kernen am Vienna Scientifc Cluster kann pro $24 \mathrm{~h}$ eine Umdrehung des Turboladerverdichters gerechnet werden (wobei eine Umdrehung aus 360 Zeitschritten besteht). Dies ergibt eine produzierte Datenmenge von etwa 1.3 TB pro Umdrehung. Aufgrund der hohen Machzahl im Verdichterlaufrad wird in der Akustiksimulation eine spezielle Randbedingung zwischen Einlass und Laufrad und Auslass und Laufrad verwendet. Diese Randbedingung erlaubt es, dass nur der Einlass und der Auslass ohne 


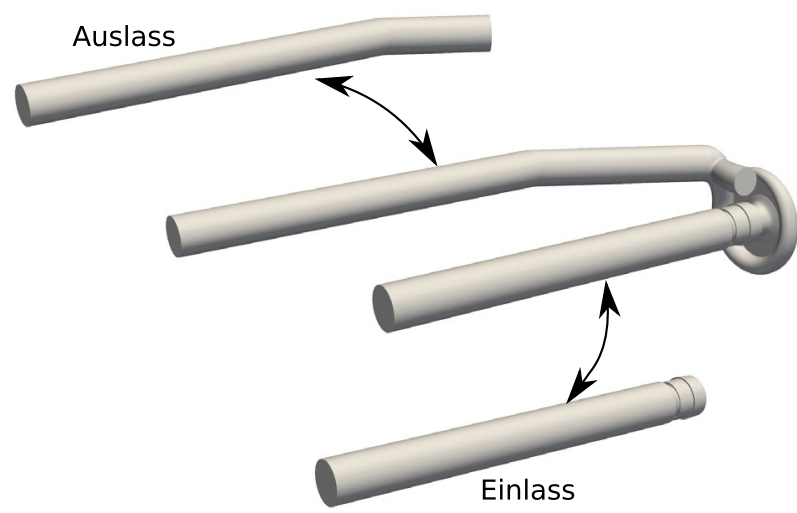

Abb. 6. Akustisches Ausbreitungsgebiet von Auslass und Einlass ohne dem Verdichterlaufrad

dem Laufrad simuliert wird und ermöglicht dadurch die Vernachlässigung der Regionen mit Überschallströmung $(M a>1)$. Durch die Vernachlässigung der rotierenden Region kann die Akustiksimulation im Frequenzbereich durchgeführt werden. Der Vorteil der Simulation im Frequenzbereich ist, dass Frequenzen die besonders interessant sind (wie z. B. die Drehzahl des Laufrades, oder die Blattfolgefrequenz) gesondert simuliert werden können. Die Simulation einzelner Frequenzen ist dabei sehr schnell. Die Akustiknetze für Einlass und Auslass bestehen jeweils aus etwa 10 Millionen Zellen. Die Zellgröße orientiert sich dabei an dem zu untersuchenden Frequenzbereich (höhere Frequenzen verlangen kleinere Zellen) und hat neben der Größe des Turboladerverdichters einen direkten Einfluss auf die Anzahl der Zellen (Abb. 6). Da für diese Untersuchung die Lighthill Analogie verwendet wird, ist das Ergebnis der Akustiksimulation eine Überlagerung von akustischem und aerodynamischem Druck. Somit lässt sich über die reine akustische Simulation lediglich eine Aussage über die Entstehungsmechanismen, sowie die räumliche Verteilung der Quellterme treffen, nicht aber über die tatsächliche Akustik innerhalb des Turboladers. Durch das Wissen der Verteilung der Quellterme ist es jedoch möglich, geometrische Optimierungen durchzuführen und alleine dadurch die Akustik zu verbessern. Weil außerdem der Beobachter, welcher den Lärm, den ein Turbolader verursacht, von außerhalb des Verdichters wahrnimmt, ist die nicht vorhandene Möglichkeit der Untersuchung vom reinen akustischen Druck innerhalb der Struktur nicht entscheidend. Durch die Möglichkeit der Kopplung von der akustischen Lighthill Simulation mit der Strukturmechanik und anschließend mit dem umgebenden Fluid, ist eine akustische Bewertung dennoch realisierbar.

\subsection{Ventilator im Kühlkreislauf von Elektromotoren}

In jedem modernen Automobil befinden sich mehrere Ventilatoren die für den Betrieb unabdingbar sind. In diesem Anwendungsbeispiel wollen wir uns dabei auf axial durchströmte Ventilatoren konzentrieren, wie sie z. B. häufig im Kühlkreislauf von Automobilen verwendet werden. Ähnliche Ventilatoren die lediglich geringere Durchmesser haben, werden dabei auch in HVAC Systemen verwendet. Der untersuchte Ventilator $[4,5]$ dreht sich dabei mit $1500 \mathrm{Um}$ drehungen pro Minute und erreicht damit eine maximale Machzahl von $M a \approx 0.113$. Das Laufrad hat einen Durchmesser von $\varnothing 495 \mathrm{~mm}$ und das Netz für die Strömungssimulation besteht in Summe aus in etwa 33 Millionen Zellen. Aufgrund der niedrigen Machzahl kann die Strömung inkompressible gerechnet und dadurch die PCWE Formulierung verwendet werden. Die Ergebnisse der Akustiksimulation beinhaltet also direkt das akustische Schallfeld. Das Akustiknetz ist dabei aus knapp über einer Million Zellen aufgebaut. Der mit

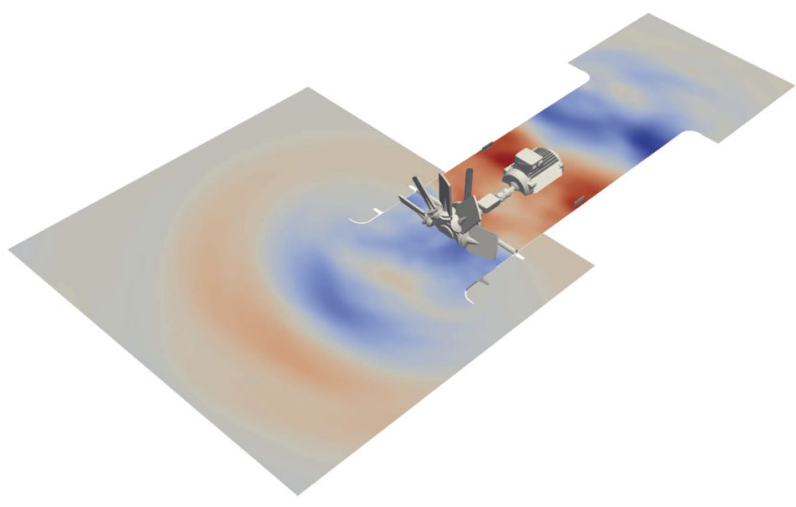

Abb. 7. Akustisches Druckfeld in einer Querschnittsdarstellung des Ventilators zu einem bestimmten Zeitpunkt der Simulation [5]



Abb. 8. Spektrum der akustischen Druckschwankungen in einem Auswertepunkt $100 \mathrm{~mm}$ entfernt vom Verdichterlaufrad, exakt auf Narbenhöhe [5]

Abstand größte Zeitaufwand dieser Untersuchung kommt der Strömungssimulation zu. Hierbei wurden auf 256 Kernen vom Vienna Scientific Cluster innerhalb von 225.5 Stunden die Quellterme von 20000 Zeitschritten exportiert, was in Summe Daten im Umfang von 32.1 TB entspricht. Für die Simulation eines Zeitschrittes der akustischen Ausbreitungsrechnung, die nach der Interpolation durchgeführt werden kann, war nur mehr 29 Sekunden auf 4 Kernen eines lokalen Rechenservers notwendig. Die dabei erzielten Simulationsergebnisse wurden mit Messungen validiert und zeigen eine gute Übereinstimmung [13]. Die Multiphysics-Software openCFS ist dabei in der Lage, das akustische Druckfeld für das gesamte Simulationsgebiet (siehe Abb. 7), sowie das Ergebnis für virtuelle Mikrophonpositionen auszugeben. Simulationsergebnisse einer bestimmten Position eignen sich um Simulations und Messergebnisse zu vergleichen (siehe Abb. 8).

\section{Resümee}

Der hybride strömungskustische Simulationsansatz bietet gleich mehrere Vorteile in der praktischen Anwendung. Durch die getrentte Simulation des Strömungs- und Akustikfeldes bietet sich die Möglichkeit, zwei unterschiedliche Rechengitter zu erstellen, die auf ihre jeweilige Anwendung optimiert sind. Darüber hinaus ermöglichen strömungsakustische Quellterme eine einfache und schnelle Optimierung, da Quellterme auf verschiedenste Akustiknetze interpoliert werden können. Durch die Möglichkeit, Simulationen unter Anwendung spezieller Randbedingungen nur für gezielte Frequenzen durchzuführen, kann der Einfluss verschiedenster geometrischer 
Feinheiten innerhalb kürzester Zeit auf z. B. bestimmte physikalische Phänomene wie die Blattfolgefrequenz untersucht werden. Abschließend lässt sich festhalten, dass die präsentierte Methodik bei verschiedensten praxisrelevanten Anwendungen zum Einsatz kommt und dabei gute Ergebnisse liefert. Aktuell wird an einer Effizienzsteigerung von openCFS gearbeitet und neue Methoden implementiert, um in Zukunft zusätzliche Funktionen zur Verfügung zu stellen.

\section{Danksagung}

Die Autoren bedanken sich bei der österreichischen Forschungsförderungsgesellschaft FFG für die Förderung im Zuge mehrerer Projekte. Im Zuge dieser Projekte wurde an unterschiedlichen Teilen des präsentierten Simulationsansatz geforscht und dieser erweitert. Außerdem bedanken wir uns bei allen Personen, die im Zuge ihrer Arbeit an der Entwicklung von openCFS beteiligt waren. Darüber hinaus bedanken wir uns bei dem Vienna Scientific Cluster für die Möglichkeit der Nutzung eines modernen Supercomputers.

Funding Note Open access funding provided by Graz University of Technology.

Hinweis des Verlags Der Verlag bleibt in Hinblick auf geografische Zuordnungen und Gebietsbezeichnungen in veröffentlichten Karten und Institutsadressen neutral.

Open Access Dieser Artikel wird unter der Creative Commons Namensnennung 4.0 International Lizenz veröffentlicht, welche die Nutzung, Vervielfältigung, Bearbeitung, Verbreitung und Wiedergabe in jeglichem Medium und Format erlaubt, sofern Sie den/die ursprünglichen Autor(en) und die Quelle ordnungsgemäß nennen, einen Link zur Creative Commons Lizenz beifügen und angeben, ob Änderungen vorgenommen wurden. Die in diesem Artikel enthaltenen Bilder und sonstiges Drittmaterial unterliegen ebenfalls der genannten Creative Commons Lizenz, sofern sich aus der Abbildungslegende nichts anderes ergibt. Sofern das betreffende Material nicht unter der genannten Creative Commons Lizenz steht und die betreffende Handlung nicht nach gesetzlichen Vorschriften erlaubt ist, ist für die oben aufgeführten Weiterverwendungen des Materials die Einwilligung des jeweiligen Rechteinhabers ein-

\section{Autoren}

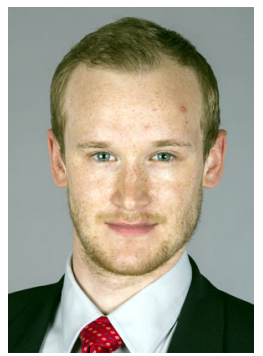

\section{Clemens Freidhager}

schloss 2018 das Studium für Maschinenbau an der TU Wien ab und arbeitete im direktem Anschluss bis Juni 2020 als Projektassistent am Institut für Mechanik und Mechatronik der TU Wien. Direkt anschließend wechselte er als Projektassistent an das Institut für Grundlagen und Theorie der Elektrotechnik an der TU Graz. Sein Forschungsschwerpunkt liegt dabei auf strömungsakustischen Simulationen für rotierende Systemen im Zuge von Forschungsprojekten die sich auf automobile Anwendung sepzifizieren.



\section{Paul Maurerlehner}

absolvierte 2020 das Masterstudium Maschinenbau an der TU Wien, wo er sich auf das Gebiet der Strömungsakustik vertiefte und in einem Forschungsprojekt über die menschliche Stimmerzeugung als Projektassistent am Institut für Mechanik und Mechatronik tätig war. Seit 2020 ist er am Institut für Grundlagen und Theorie der Elektrotechnik an der TU Graz im Rahmen einer Dissertation im Be- zuholen. Weitere Details zur Lizenz entnehmen Sie bitte der Lizenzinformation auf http://creativecommons.org/licenses/by/4.0/deed.de.

\section{Literatur}

1. Schoder, S., Junger, C., Kaltenbacher, M. (2020): Computational aeroacoustics of the EAA benchmark case of an axial fan. Acta Acust., 4, 22.

2. Kaltenbacher, M. (2015): Numerical simulation of mechatronic sensors und actuators - finite elements for computational multiphysics. 3. ed. Berlin: Springer.

3. Schoder, S., Roppert, K., Weitz, M., Junger, C., Kaltenbacher, M. (2020): Aeroacoustic source term computation based on radial basis functions. International Journal for Numerical Methods in Engineering, 121, 2051-2067.

4. Junger, C. (2019): Computational aeroacoustics for the characterization of noise sources in rotating systems. PhD Thesis.

5. Wurzinger, A. (2020): Aeroacoustic simulation of flow parts in medical and automotive applications. Master Thesis.

6. Golloch, R. (2005): Downsizing bei Verbrennungsmotoren, Ein Wirkungsvolles Konzept zur Kraftstoffverbrauchssenkung. Berlin: Springer.

7. Nguyen-Schäfer, H. (2013): Aero and vibroacoustics of automotive turbochargers. Berlin: Springer.

8. Hüppe, A., Grabinger, J., Kaltenbacher, M., Reppenhagen, A., Dutzler, G., Kühnel, W. (2014): 20th AIAA/CEAS aeroacoustics conference (S. 2739)

9. Lighthill, M. J. (1952): On sound generated aerodynamically I. General theory. In Proceedings of the royal society of London. Series A. Mathematical and physical sciences (S. 564-587).

10. Lighthill, M. J. (1954): On sound generated aerodynamically II. Turbulence as a source of sound. In Proceedings of the royal society of London. Series A. Mathematical and physical sciences (S. 1-32).

11. Schoder, S., Kaltenbacher, M. (2019): Hybrid aeroacoustic computations: state of art and new achievements. Journal of Theoretical and Computational Acoustics, 27. 1950020

12. VSC - Vienna Scientific Cluster, Operngasse 111040 Wien, Austria

13. Schoder, S., Wurzinger, A., Junger, C., Weitz, M., Freidhager, C., Roppert, K., Kaltenbacher, M. (2021): Application limits of conservative source interpolation methods using a low Mach number hybrid aeroacoustic workflow. Journal of Theoretical and Computational Acoustics, 29, 2050032

14. openCFS, Finite Elements for Computational Multiphysics, www.opencfs.org.

15. Schoder, S., Junger, C., Weitz, M., Kaltenbacher, M. (2019): Conservative source term interpolation for hybrid aeroacoustic computations. In 25th AIAA/CEAS aeroacoustics conference. reich der Strömungs- und Vibroakustik angestellt. Dabei liegt sein Forschungsschwerpunkt auf der Schallentstehung und -abstrahlung von Rohrströmungen.

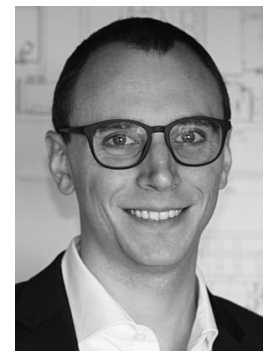

Klaus Roppert

recieved his Doctoral degree from TU Wien in 2020. Since 2020, he holds a PostDoc position in the Multiphysics group at TU Graz. His research focuses mainly on the development of innovative finite element methods for various coupled physical problems, with a strong focus on electromagnetics. This includes simulation methodologies for induction heating processes, hysteretic material behavior, power electronics and aeroacoustic problems, just to name a few. 


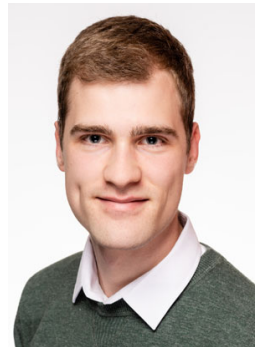

\section{Andreas Wurzinger}

Schloss 2019 sein Bachelorstudium und 2020 sein Masterstudium in Maschinenbau an der Technischen Universität Wien ab. Während dem Studium arbeitete er als studentischer Mitarbeiter an Forschungsprojekten im Bereich Regelungstechnik und Strömungsakustik. 2021 startete er sein Doktoratsstudium an der Technischen Universität Graz als Projektassistent am Institut für Grundlagen und Theorie der Elektrotechnik. Seine Forschungsschwerpunkte konzentrieren sich dabei auf die numerische Simulation von strömungsakustischen Problemstellungen.

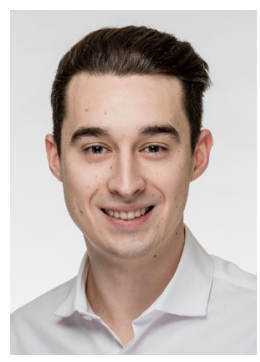

\section{Alexander Hauser}

Schloss 2019 das Bachelorstudium Wirtschaftsingenieurwesen-Maschinenbau an der TU Wien ab und befindet sich aktuell im Masterstudium. In seiner Bachelorarbeit beschäftigte er sich mit der numerischen Simulation der menschlichen Stimmgebung. Seit 2019 ist er studentischer Projektmitarbeiter am Institut für Mechanik und Mechatronik an der TU Wien und unterstützt die Forschungsgruppe für Strömungsakustik.

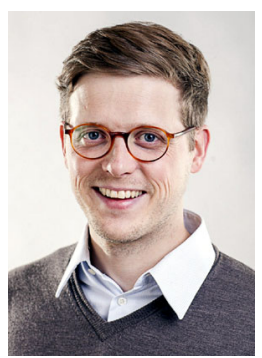

\section{Martin Heinisch}

obtained his Dipl.-Ing. (M.Sc.) in mechatronics from Johannes Kepler University Linz, Austria, in 2009. After his Master studies he went to the University of California, Los Angeles (U.C.L.A.) as a Marshall Plan Scholarship grantee, where he did research in the field of microfluidic applications and self assembling systems. In 2015 he finished an International Joint Docotorate at the Institute for Microelectronics and Microsensors at the Johannes Kepler University Linz, Austria and the Université de Bordeaux, France on resonating liquid sensors. Since 2016, he is working in the field of Turbocharger Acoustics.

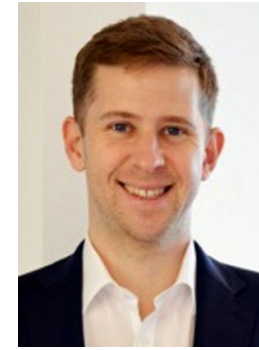

\section{Stefan Schoder}

received his Doctoral degree from TU Wien in 2019. Since 2020, he has been the head of Research Group Vibro- and Aeroacoustics and Ass.-Prof. in the field of Aeroacoustics at TU Graz. His research is unit develops advanced computational algorithms for coupled field problems technical and medical applications. This includes vibroacoustic and aeroacoustic applications, advanced finite element methods, and source term filtering techniques with mesh-less methods.

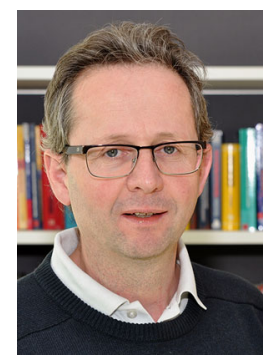

\section{Manfred Kaltenbacher}

received his Dipl.-Ing. in electrical engineering from the Technical University of Graz, Austria in 1992, his Ph.D. in technical science from the Johannes Kepler University of Linz, Austria in 1996, and his habilitation from Friedrich-Alexander-University of ErlangenNuremberg, Germany, in 2004. In 2008 he became a full professor for Applied Mechatronics at Alps-Adriatic University Klagenfurt, Austria. In 2012 he moved to Vienna University of Technology, Austria, as a full professor for Measurement and Actuator Technology, and in 2020 he became the head of the Institute of Fundamentals and Theory in Electrical Engineering at TU Graz, Austria. His research involves theory, modeling, simulation and experimental investigation of complex systems in engineering, material and medical science. A main focus is on the development of advanced Finite Element (FE) methods for multi-field problems (electromagnetics-mechanics, mechanics-acoustics, piezoelectricity, flow dynamics - mechanics, aeroacoustics), and their application to design mechatronic sensors and actuators. 